This is the submitted version of the article:

Masvidal-Codina E., Illa X., Dasilva M., Calia A.B., Dragojevic T., Vidal-Rosas E.E., Prats-Alfonso E., Martínez-Aguilar J., De la Cruz J.M., Garcia-Cortadella R., Godignon P., Rius G., Camassa A., Del Corro E., Bousquet J., Hébert C., Durduran T., Villa . High-resolution mapping of infraslow cortical brain activity enabled by graphene microtransistors. Nature Materials, (2019). 18. : 280 - . 10.1038/s41563-018-0249-4.

Available at: https://dx.doi.org/10.1038/s41563-018-0249-4 


\section{High-resolution mapping of infraslow cortical brain activity enabled by graphene microtransistors}

Eduard Masvidal-Codina ${ }^{1}$, Xavi Illa ${ }^{2,1}$, Miguel Dasilva ${ }^{3}$, Andrea Bonaccini Calia ${ }^{4}$ Tanja Dragojević $^{5}$, Ernesto E. Vidal-Rosas ${ }^{5}$, Elisabet Prats-Alfonso ${ }^{2,1}$, Javier Martínez-Aguilar ${ }^{1,2}$, Jose M. De la Cruz Sanchez ${ }^{4}$, Philippe Godignon ${ }^{1}$, Gemma Rius ${ }^{1}$, Alessandra Camassa ${ }^{3}$, Elena Del Corro ${ }^{4}$, Jessica Bousquet ${ }^{4}$, Clement Hébert ${ }^{4}$, Turgut Durduran ${ }^{5,6}$, Rosa Villa ${ }^{1,2}$, Maria V Sanchez-Vives ${ }^{3,6}$, Jose A Garrido ${ }^{4,6 *}$ and Anton Guimerà-Brunet ${ }^{1,2 *}$

1 Institut de Microelectrònica de Barcelona, IMB-CNM (CSIC), Esfera UAB, Bellaterra, Spain

2 Centro de Investigación Biomédica en Red en Bioingeniería, Biomateriales y Nanomedicina (CIBER-BBN), Madrid, Spain

3 Institut d'Investigacions Biomèdiques August Pi i Sunyer (IDIBAPS), Barcelona, Spain

4 Catalan Institute of Nanoscience and Nanotechnology (ICN2), CSIC and The Barcelona Institute of Science and Technology (BIST), Campus UAB, Bellaterra, Barcelona, Spain

5 ICFO-Institut de Ciéncies Fotòniques, The Barcelona Institute of Science and Technology, Castelldefels, Barcelona, Spain

6 Institució Catalana de Recerca i Estudis Avançats (ICREA), Barcelona, Spain

* Correspondence:

anton.guimera@imb-cnm.csic.es; joseantonio.garrido@icn2.cat

Keywords: cortical spreading depression, DC-ECoG, graphene, infraslow. 


\section{Abstract}

2 Recording infraslow brain signals $(<0.1 \mathrm{~Hz})$ with microelectrodes is severely hampered by current 3 electrode materials, primarily due to limitations resulting from voltage drift and high electrode 4 impedance. Hence, most recording systems include high-pass filters that solve saturation issues but 5 come in hand with loss of physiological and pathological information. In this work we use for the 6 first time flexible epicortical and intracortical arrays of graphene solution-gated field-effect 7 transistors (gSGFETs) to map cortical spreading depression in rats and demonstrate that gSGFETs 8 are able to record infraslow signals alongside with signals in the typical local field potential

9 bandwidth. This capability results from the direct field-effect coupling of the active transistor, in 10 contrast to standard passive electrodes, as well as from the electrochemical inertness of graphene. 11 Taking advantage of such functionality, we envision broad applications of gSGFET technology for 12 monitoring infraslow brain activity both in research and in the clinic. 
13 Recently, there has been a particular resurgence of interest in fluctuations of brain activity occurring 14 at $<0.1 \mathrm{~Hz}$, commonly referred to as very slow, ultraslow or infraslow activity (ISA) ${ }^{1}$. ISA is 15 suggested to have a unique neurophysiological basis ${ }^{2}$, and to be indicative of brain states (e.g. sleep, 16 anesthesia, coma, wakefulness $)^{2-4}$. ISA is also correlated with resting-state networks in functional 17 magnetic resonance imaging ${ }^{5}$ and may significantly contribute to the high variability observed in the 18 time course of physiological signals ${ }^{6,7}$. Interestingly, spreading depolarizations ${ }^{8}$, and more 19 specifically, cortical spreading depression (CSD), occur at infralow frequencies. CSD is defined as a 20 slowly propagating wave of near-complete depolarization of neurons and astrocytes followed by a 21 period of electrical activity suppression. CSD is often triggered in individuals suffering stroke or 22 brain injury as well as migraines and recent research has shown that CSDs play a significant role in 23 brain pathophysiology ${ }^{9-11}$. Therefore, monitoring ISA can be very valuable for clinical diagnosis, 24 prognosis and therapy in neurocritical care ${ }^{12-14}$.

25 Non-invasive techniques such as electroencephalography (EEG) and magnetoencephalography 26 (MEG) have been used to study ISA ${ }^{15,16}$. However, their limited spatial resolution, and averaged 27 signal impose serious limitations, e.g. EEG alone is not sufficient for non-invasive CSD 28 detection $^{13,17}$. Hence, invasive electrophysiological techniques are the most widely used to record 29 infraslow brainwaves. The proper recording of ISA requires the use of direct-coupled amplifiers and 30 extremely stable and low-impedance invasive electrodes. Traditionally, liquid-filled glass 31 micropipettes are used, which allow only one or few-point measurements ${ }^{18}$. For higher spatial 32 resolution and mapping, non-polarizable silver/silver chloride $(\mathrm{Ag} / \mathrm{AgCl})$ electrodes could be used, 33 which prevent charge accumulation at the interface and therefore voltage drift. However, due to the 34 toxicity of silver, the use of such electrodes for human or chronic animal in vivo monitoring is not an 35 option $^{19}$. This has fostered the search for alternative microelectrode materials with low impedance 36 and drift although none has yet been found capable of offering comparable performance as $\mathrm{Ag} / \mathrm{AgCl}$ 37 electrodes ${ }^{20}$. So, ISA recordings in humans are currently performed with platinum electrodes, which 38 challenge CSD detection due to artifacts and transients ${ }^{21}$. Furthermore, as is discussed in this work, 39 miniaturization of electrode size to achieve higher spatial resolution causes intrinsic high-pass 40 filtering of ISA due to the associated electrode impedance increase ${ }^{22,23}$. Invasive optical techniques 41 such as calcium imaging are also used to monitor ISA, but still nowadays have serious challenges to 42 resolve high-frequency activity for a large number of neurons ${ }^{24,25}$ and their intrinsic need of 43 indicators limits the translation to the clinics. Therefore, a technique which allows for measuring 44 large-scale, high-spatiotemporal resolution recordings including infraslow frequencies in a 45 potentially fully implantable, nontoxic, clinical-scale system is still missing (Table S1).

46 Alternatively to the commonly used microelectrode technology, recording electrophysiological 47 signals with field-effect transistors (FET) offers several advantages including that they are less 48 sensitive to environmental noise thanks to their intrinsic voltage-to-current amplification, and that 49 they can be easily multiplexed ${ }^{26}$. Nonetheless, the difficulties to combine high gate capacitance and 50 carrier mobility silicon FETs with flexible materials has historically hampered its use for in vivo 51 recordings $^{27}$. Graphene solution-gated field-effect transistors (gSGFETs) have been proposed to 52 potentially overcome all previous drawbacks at once ${ }^{28}$. The two-dimensional nature of graphene 53 provides the highest surface-to-volume ratio possible, making graphene very sensitive to charges at 
54 its surface; further, its flexibility allows gSGFETs to be embedded in ultra-soft and flexible 55 substrates without loss of performance ${ }^{29}$. Moreover, the wide electrochemical window and 56 biocompatibility of graphene allows direct contact with biological fluids and tissues and ensures a 57 safe operation in in vivo conditions ${ }^{30}$. Taking advantage of the above-mentioned properties, in 58 previous works, we demonstrated that gSGFETs are able to record local field potentials ${ }^{31,32}$.

59 In this work we investigate the potential of graphene microtransistors to record infraslow brain 60 activity by performing in vivo recordings where we use, for the first time, gSGFETs for both 61 epicortical and intracortical mapping of cortical spreading depression. We found that graphene 62 microtransistors are excellent devices for recording infraslow signals and, furthermore, they do not 63 compromise the acquisition of signals in the conventional local field potential bandwidth. We also 64 demonstrate that gSGFET technology can be used in combination with optical techniques, such as 65 laser speckle contrast imaging, to obtain 2-D maps of the neurovascular coupling.

\section{Structure, fabrication and characterization of gSGFET arrays}

67 A gSGFETS is a device in which graphene is used as channel material, contacted by two metal leads 68 (source and drain terminals), and is immersed in an electrolyte solution where a reference electrode is 69 used as gate terminal (Fig. 1a). We fabricated flexible probes containing arrays of gSGFETs in both 70 epicortical and intracortical designs. In particular, a 4x4 array of $100 \mu \mathrm{m}$ wide by $50 \mu \mathrm{m}$ long

71 graphene channels was designed for epicortical recordings while a design consisting of a linear array 72 of 15 graphene channels ( $80 \mu \mathrm{m}$ width, $30 \mu \mathrm{m}$ length) was used for intracortical recordings (Fig. 1b).

73 Both array designs were fabricated on a $10 \mu \mathrm{m}$ thick polyimide layer coated on a 4-inch silicon wafer 74 using the process previously reported in Hébert et al. ${ }^{32}$. Flexible gSGFET arrays were placed in zero 75 insertion force connectors for interfacing with recording electronics (Fig. 1c). The transfer curve, 76 drain current $\left(I_{d s}\right)$ vs gate-source voltage $\left(V_{g s}\right)$, of all gSGFETs in each array was measured with a 77 fixed drain-source voltage $\left(V_{d s}\right)$. The dispersion of the charge neutrality point $(\mathrm{CNP}=243.6 \pm 6.1$ $78 \mathrm{mV}$ ), which is the minimum of the transfer curve, indicates the homogeneity of the transistors (Fig. 79 1d). Importantly, since the $V_{g s}$ and $V_{d s}$ bias are shared, the small CNP dispersion allows near-optimal 80 recording performance for all gSGFETs in the same array. Figure 1e shows the sum of leakage 81 current $\left(I_{g s}\right)$ for all gSGFETs in the array, which is in the nA range throughout the voltage sweep, 82 demonstrating the good insulation of the passivation layer and the negligible reactivity of the

83 graphene. Furthermore, we measured the frequency response of the transconductance $\left(\mathrm{g}_{\mathrm{m}}\right)$ of a 84 gSGFET, which indicates the efficiency of the signal coupling $\left(\partial I_{d s} / \partial V_{g s}\right)$, obtaining constant values 85 in a wide bandwidth including inflalow frequencies (Fig. 1f-g). The negative $g_{m}$ for $V_{g s}$ values lower 86 than the CNP results in an inversion ( $180^{\circ}$ phase) of the signals measured at such bias; for $\mathrm{V}_{\mathrm{gs}}$ values 87 higher than the CNP the signal phase is preserved. 
a
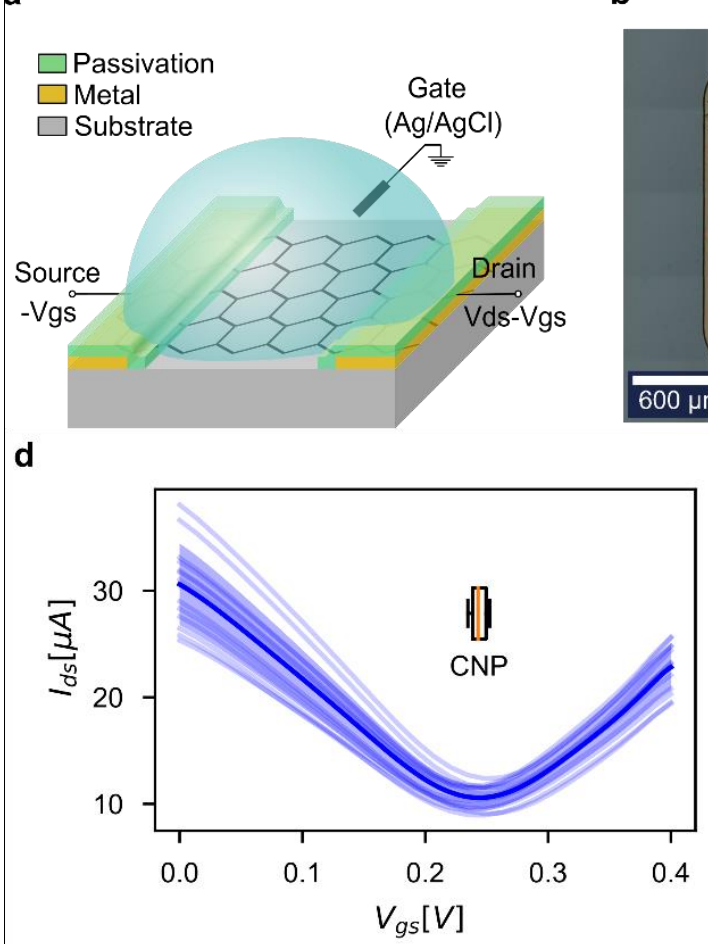

f

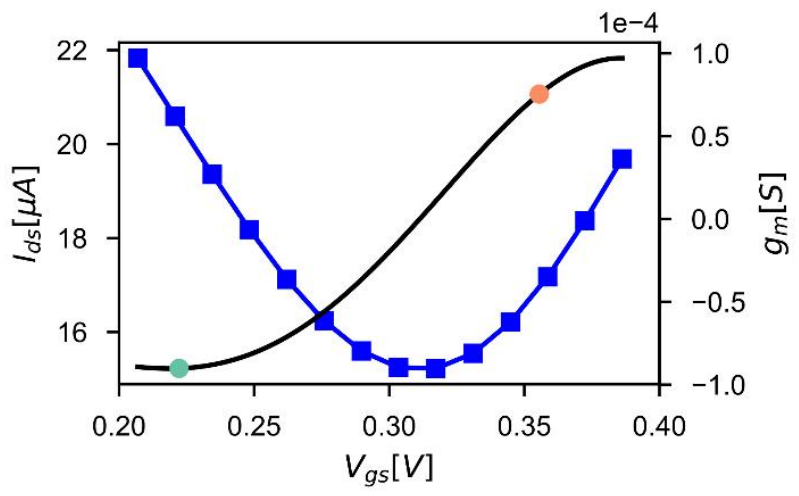

b

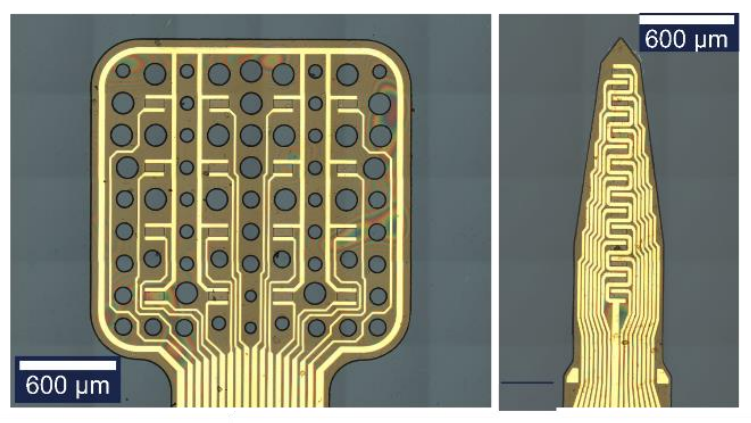

e

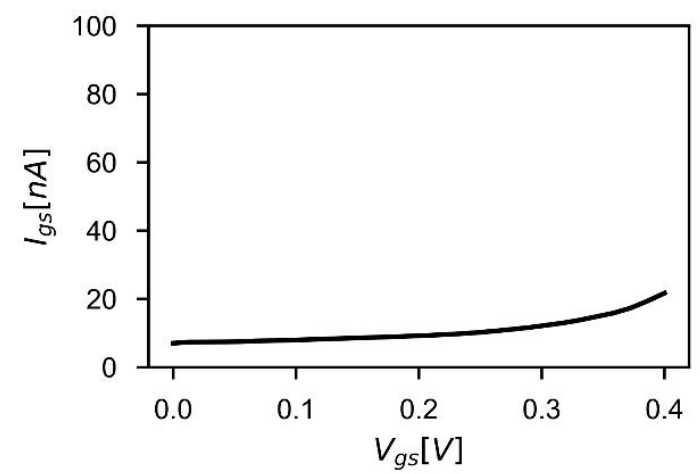

g

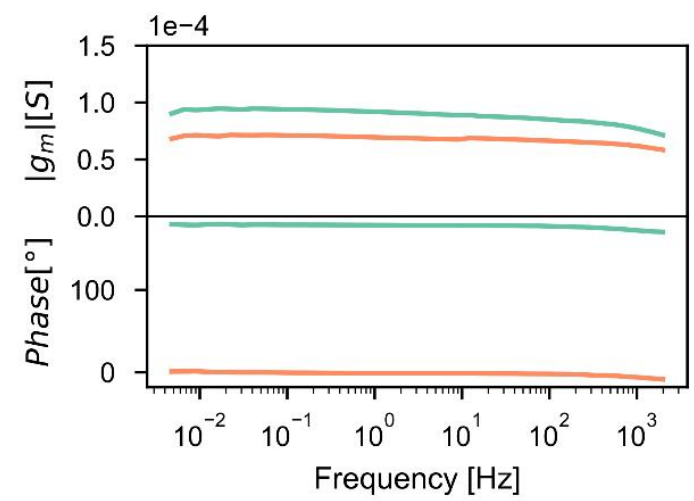

c

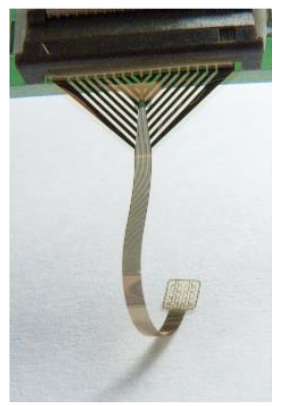
8

Fig. 1 | Flexible graphene solution-gated field-effect transistor array technology and characterization. a, Schematic of a graphene transistor polarized in common gate mode. b, Optical microscope images of the active area of the $4 \times 4$ gSGFET array and the 15 channel intracortical array. c, Photograph of the neural probe after peeling from the wafer and being introduced into a zero insertion force connector. d-g, Steady-state and frequency response characterization of a $100 \times 50-\mu \mathrm{m}^{2}$ gSGFET array in $10 \mathrm{mM}$ phosphate buffered saline (PBS) and with a drain-source voltage bias $\left(V_{d s}\right)$ of $50 \mathrm{mV}$. d, gSGFET transfer curves (blue lines), drainsource current $\left(I_{d s}\right) v s$ gate-source voltage $\left(V_{g s}\right)$, together with the mean (dark blue) and standard deviation (blue shade). Boxplot inset shows charge neutrality point dispersion. e, Leakage current $\left(I_{g s}\right)$ of all gSGFETs in the array. f, Transfer curve (blue squares and line) and its first derivative (transconductance $\left(g_{m}\right)$, black line) of a gSGFET. g, Frequency response of the transconductance at two different points of the transfer curve (e): $V_{g s}$ lower than the CNP (green), where $\mathrm{g}_{\mathrm{m}}$ is negative resulting in a signal inversion $\left(180^{\circ}\right.$ phase); and $V_{g s}$ higher than the CNP (orange), where $\mathrm{g}_{\mathrm{m}}$ is positive and thus results in no inversion $\left(0^{\circ}\right.$ phase). Independently of the branch of the transfer curve where a gSGFET is polarized, the module of $\mathrm{g}_{\mathrm{m}}$ is similar to the steady-state value for a wide bandwidth $(\approx 0-1 \mathrm{kHz})$. 

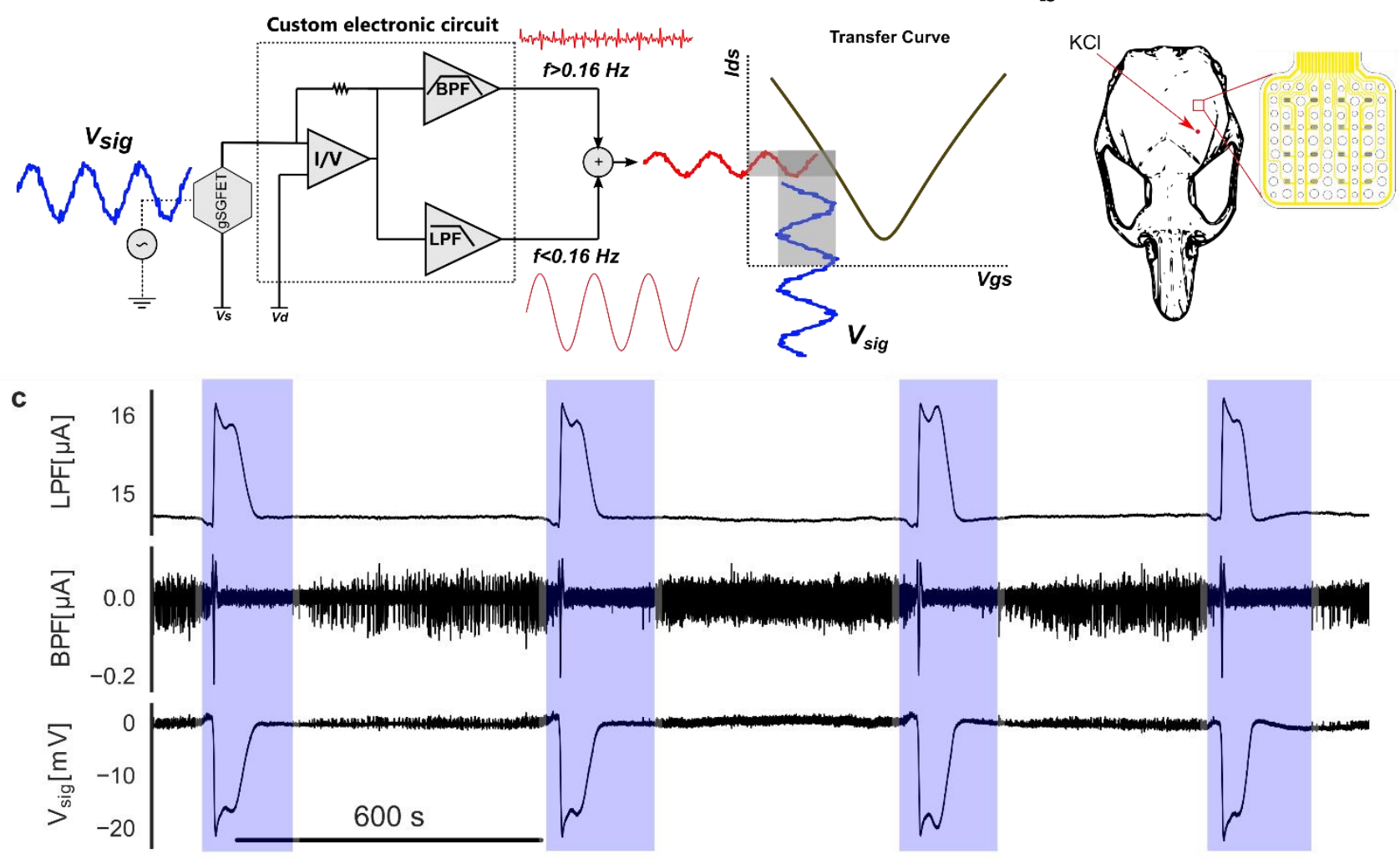

d

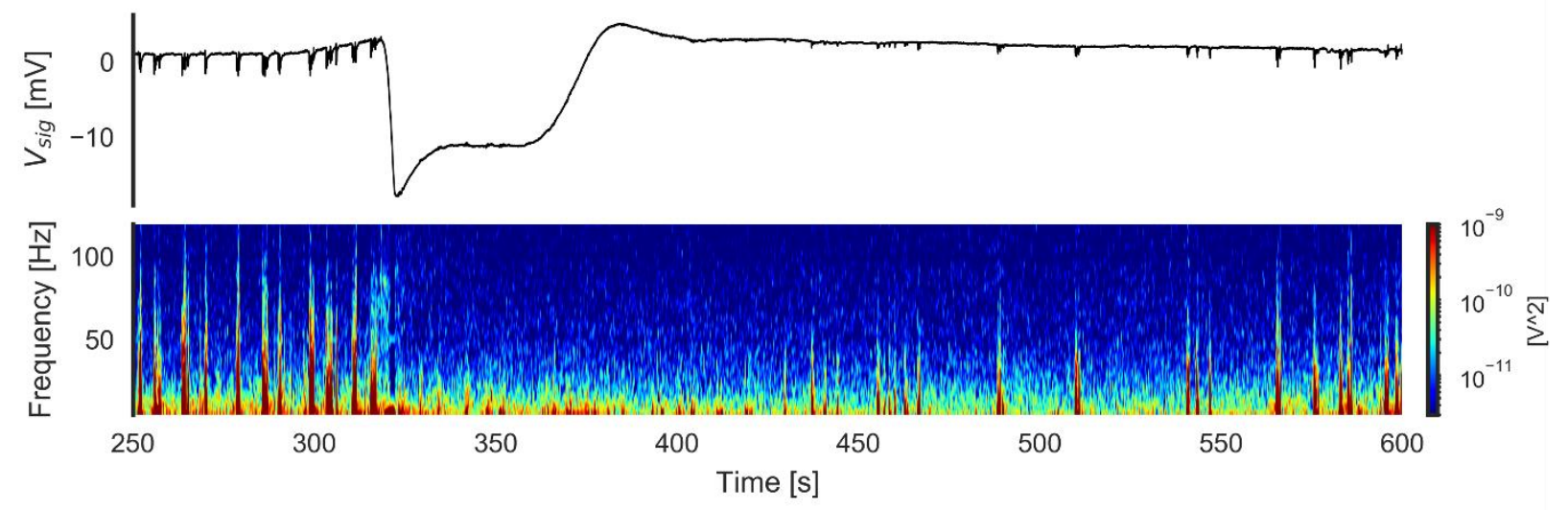

105 Fig. 2 | Infraslow, local field potential, and wide-band in vivo gSGFET recordings of cortical spreading 106 depression (CSD). a, Schematic of the gSGFET recording setup and signal post processing methodology. The 107 custom electronic circuit is used to perform the in vivo characterization (transfer curve) and record the 108 transistor current in the low-pass-filtered (LPF) band and the band-pass-filtered (BPF) band. From the 109 combination of both signals and taking into account the current-to-voltage conversion, the wide-band signal $110\left(V_{s i g}\right)$ is obtained. b, Schematic of the location of the gSGFET array and the frontal craniotomy where $5 \mathrm{mM}$ $111 \mathrm{KCl}$ was applied to induce CSDs. c, Electrophysiological recordings obtained with a gSGFET epicortical array 112 during the induction of four CSD events (blue shade). From top to bottom: current LPF signal, current BPF

113 and voltage-converted wide-band signal. d, Voltage-converted wide-band signal of a CSD event and 114 spectrogram showing the silencing of activity. 


\section{In vivo wide-band recordings}

118 Cortical spreading depression ${ }^{9,11,18}$ was chosen to illustrate the capabilities of graphene transistors to 119 record in a wide bandwidth. Experimentally, two craniotomies were performed over the left 120 hemisphere of isoflurane-anaesthetized Wistar rats: a larger craniotomy over the primary 121 somatosensory cortex, where the epicortical probe was placed, and a smaller one in the frontal cortex, 122 where $5 \mathrm{mM} \mathrm{KCl}$ was applied locally to induce CSD (Fig. 2b). A custom electronic circuit allowed 123 us to simultaneously record at two frequency bands: low-pass filtered band (LPF, $\approx 0-0.16 \mathrm{~Hz}$ ) and 124 band-pass filtered band (BPF, $0.16 \mathrm{~Hz}-10 \mathrm{kHz})$ with different gains $\left(10^{4}\right.$, and $10^{6}$ respectively) to 125 avoid amplifier saturation due to the high-amplitude CSD signal. In a first set of experiments, we 126 recorded the LPF and BPF current signals with an epicortical gSGFET array during the induction of 127 CSD events (Fig. 2c). The graphene transistors were polarized in the hole conduction regime, i.e. $V_{g s}$

$128<\mathrm{CNP}$ (negative $\mathrm{g}_{\mathrm{m}}$ ); therefore, the recorded LPF and BPF current signals are inverted with respect 129 to the voltage signal occurring at the gate. The LPF signal shows the very slow CSD event whereas 130 the BPF signal corresponds to the local field potential, revealing the silencing of activity typical of 131 cortical spreading depression. After summation of the LPF and BPF signals and then transforming 132 the current into a voltage signal (using the transistor transfer curve $I_{d s}-V_{g s}$ recorded in vivo prior to 133 the start of the recordings), the wide-band electrophysiological signal can be obtained (see Fig. 2 a, 134 c). In each CSD event a small positive shift of $1-2 \mathrm{mV}$ generally precedes the depression, 135 immediately after which a steep negative change $(\approx-20 \mathrm{mV})$ can be observed, which slowly recovers 136 during the next minute or so. The CSD-associated silencing of high-frequency activity and its 137 progressive recovery is shown in the voltage wave and spectrogram of Fig. 2d.

\section{SGFETs $v \boldsymbol{s}$ microelectrodes: comparison of ISA recording capabilities}

139 A second set of experiments was designed to compare the performance of gSGFETs with 140 microelectrodes in in-vivo direct-coupled recordings. CSD was induced and simultaneously recorded 141 with an gSGFET epicortical array located more posterior to a neural probe containing groups of 142 triodes of $50 \mu \mathrm{m}$ diameter gold microelectrodes in which one microelectrode of each triode was 143 modified by deposition of platinum black to lower its impedance (Fig. S2). Fig. 3a shows that gold 144 and platinum black recordings exhibit very large and diverse baseline offsets as well as oscillations 145 and drifts $(-7.9 \pm 3.3$ and $-3.6 \pm 1.6 \mathrm{mV} / \mathrm{h})$, while the gSGFET signals are very stable $(1.1 \pm 1.0$ $146 \mathrm{mV} / \mathrm{h})$. Importantly, gSGFETs record significantly higher amplitude for the CSD events (-13.3 \pm 1.8 $147 \mathrm{mV})$ in comparison with gold $(-4.7 \pm 1.6 \mathrm{mV})$ and platinum black $(-3.0 \pm 0.7 \mathrm{mV})$ microelectrodes. 148 Figure $3 \mathrm{~b}$ highlights these two intrinsic limitations of microelectrode technology for the measurement 149 of ISA: polarization-induced drift and signal filtering ${ }^{20,23}$. 
a

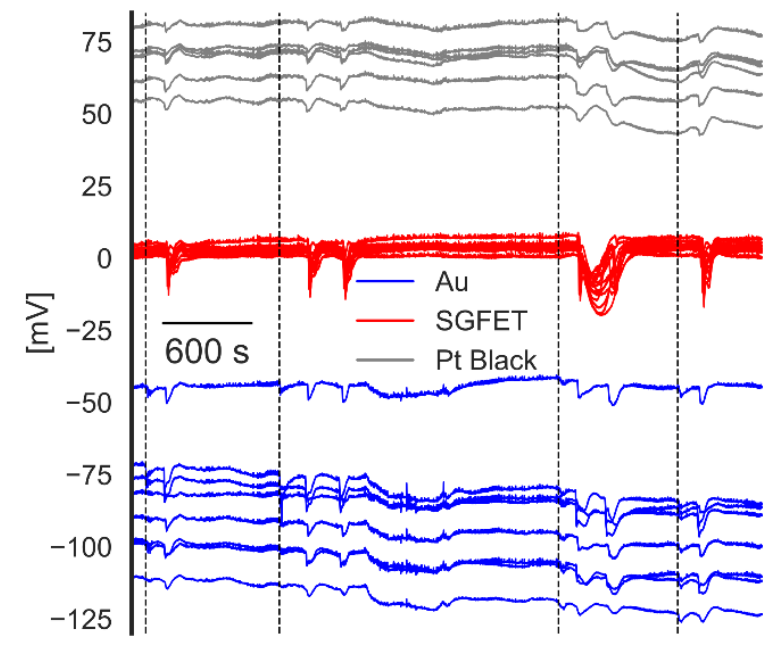

b

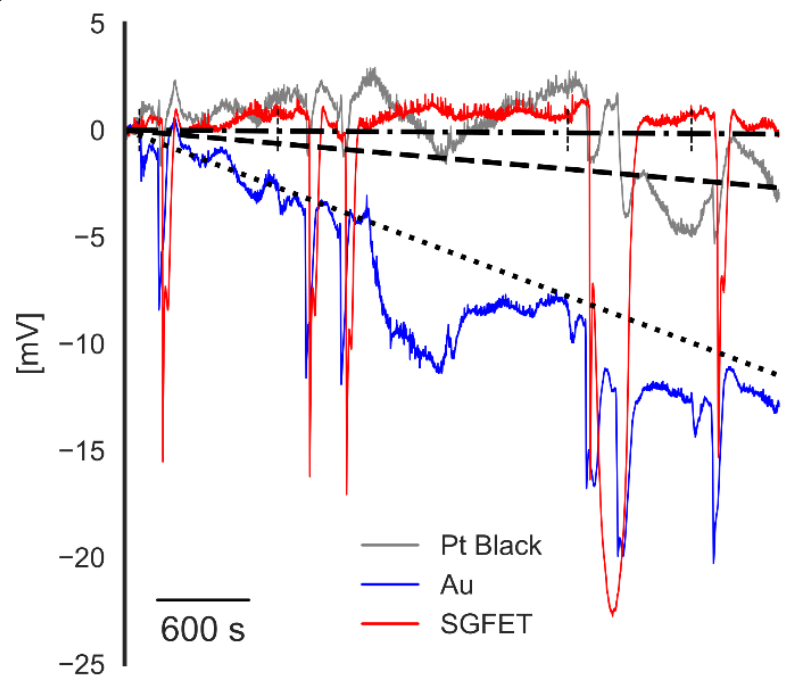

C
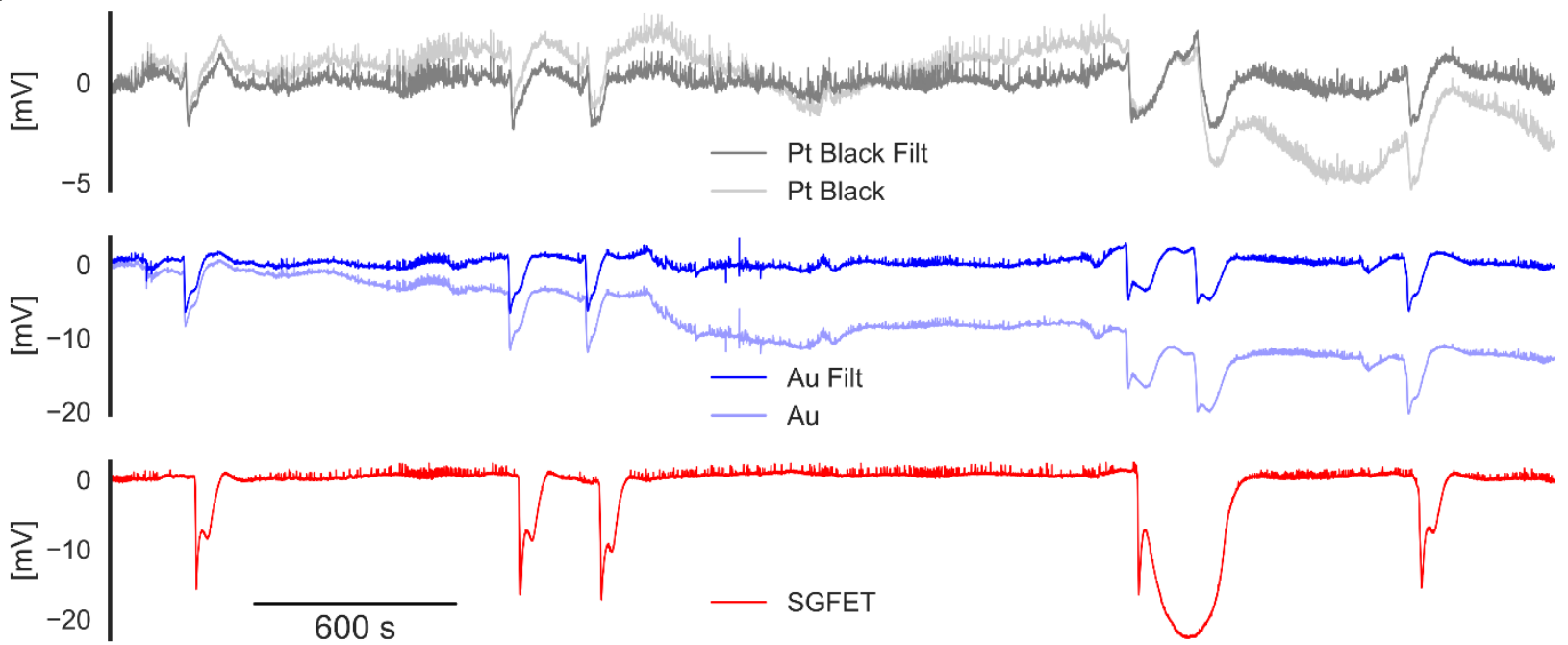

152 Fig. 3 | Comparison of gSGFET and microelectrode recordings of cortical spreading depressions. a, 153 Direct-coupled recordings of 100x $50 \mu \mathrm{m}^{2}$ gSGFET transistors and gold and platinum black $50 \mu \mathrm{m}$ diameter 154 microelectrodes. The vertical dashed lines show the time when $\mathrm{KCl}(5 \mathrm{mM})$ was applied to induce a CSD. b, 155 DC-offset removed recordings of a representative channel of each type. Black lines illustrate the mean drift: 156 dotted and dashed correspond to gold and platinum black microelectrodes, respectively, and the dash-dotted 157 line corresponds to gSGFETs. c, DC-offset removed recordings of a representative channel of each class and 158 the same signal filtered at $0.002 \mathrm{~Hz}$ to remove oscillations and drift; the gSGFET signal does not require any 159 filtering.

160 The drift of the baseline potential superimposed over the huge voltage offsets is problematic as it can 161 lead to saturation of the amplifiers used to record the signal. For this reason most microelectrode 162 recording instrumentation include a high-pass filter. More importantly, baseline drift in the form of 163 baseline oscillations in the infralow frequencies, hamper the determination of CSD characteristics 164 such as amplitude or waveform as any high-pass filter used to remove such effects will alter the 165 signal shape (see Fig.3c and Fig. S3). Another intrinsic limitation of microelectrode technology is 166 based on the relation between the microelectrode impedance and the input impedance of the 
167 recording equipment $\left(Z_{e}^{\prime}\right.$ and $Z_{a}^{\prime}$, respectively). The recorded signal $\left(V_{i n}\right)$ is determined by the 168 voltage divider formed by both impedances:

$$
V_{\text {in }}(f)=I(f) Z_{a}^{\prime}(f)=\frac{V_{\text {sig }}(f) Z_{a}^{\prime}(f)}{Z_{a}^{\prime}(f)+Z_{e}^{\prime}(f)}
$$

169 Eq. (1) implies that when $Z_{a}^{\prime}$, is not substantially larger than $Z_{e}^{\prime}$, the recorded signals will be 170 attenuated and delayed with respect to $V_{\text {sig }}{ }^{22}$. By measuring the impedance of both electrode types 171 and modelling the preamplifier impedance with the values reported by the manufacturer, we obtained 172 the voltage gain $\left(V_{\text {in }} / V_{\text {sig }}\right)$ of the equivalent circuit formed by the recording electrode and the 173 amplifier, see Fig. 4a-b. For $50 \mu \mathrm{m}$ diameter gold microelectrodes, an attenuation lower than $50 \%$ is 174 expected which is in agreement with the experimental results. For platinum black we attribute the 175 higher attenuation than predicted to electrochemical processes that impact the electrode response at 176 very low frequencies. It is important to highlight that the $Z_{a}^{\prime}>>Z_{e}^{\prime}$ requirement to achieve a voltage 177 gain equal to 1 is compromised when the electrode area is scaled down, due to the inverse relation 178 between electrode impedance and its area leading to high-pass filtering of the recorded signals.

179 In contrast, the results of the in vivo comparison provide evidence that gSGFETs are able to record 180 signals in a wide bandwidth, which we assign to the following main reasons. First, graphene exhibits 181 an excellent DC stability, as demonstrated by low in vivo drift. We attribute this to the low density of 182 states of pristine graphene near the Fermi level, which decreases the overall electronic overlap with 183 redox species ${ }^{33}$, and to the low density of extrinsic electron transfer sites, i.e. defects and edges, all 184 contributing to the excellent electrochemical inertness of CVD graphene ${ }^{24,34,35}$. The low leakage 185 current measured (Fig. 1e) also supports the electrochemical inertness. The second reason for which 186 graphene microtransistors can record infraslow signals is related to its working mechanism, which is 187 significantly different from that of electrodes. In gSGFETs, voltage oscillations near the active 188 graphene channel modulate the current flow along it (see schematic and small-signal model in Fig. 189 4a). Eq. 2 shows the relation between the recorded current $\left(\mathrm{I}_{\mathrm{ds}-\mathrm{rec}}\right)$ and the signal $\left(\mathrm{V}_{\mathrm{sig}}\right)$ :

$$
I_{d s-r e c}\left(V_{g s}, V_{s i g}\right)=I_{d s}\left(V_{g s}\right)+i_{d s}\left(V_{g s}, V_{s i g}\right)=I_{d s}\left(V_{g s}\right)+g_{m}\left(V_{g s}+V_{s i g}\right) V_{s i g}
$$

190 where $\mathrm{I}_{\mathrm{ds}}$ is the current at the bias point $\mathrm{V}_{\mathrm{gs}}$ and $\mathrm{i}_{\mathrm{ds}}$ the current variation induced by the gate signal. 191 This equation is valid and frequency-independent as long as $g_{m}$ is also frequency-independent. We 192 had previously reported that gSGFETs exhibit a transconductance that is independent of frequency 193 over the local field potential bandwidth ${ }^{32}$. In this work (Fig. 1g), we have confirmed that the 194 transconductance of gSGFETs remains constant down to infralow frequencies. Importantly, scaling 195 down the size of a transistor does not result in a decrease of $g_{m}$ at infralow frequencies. 

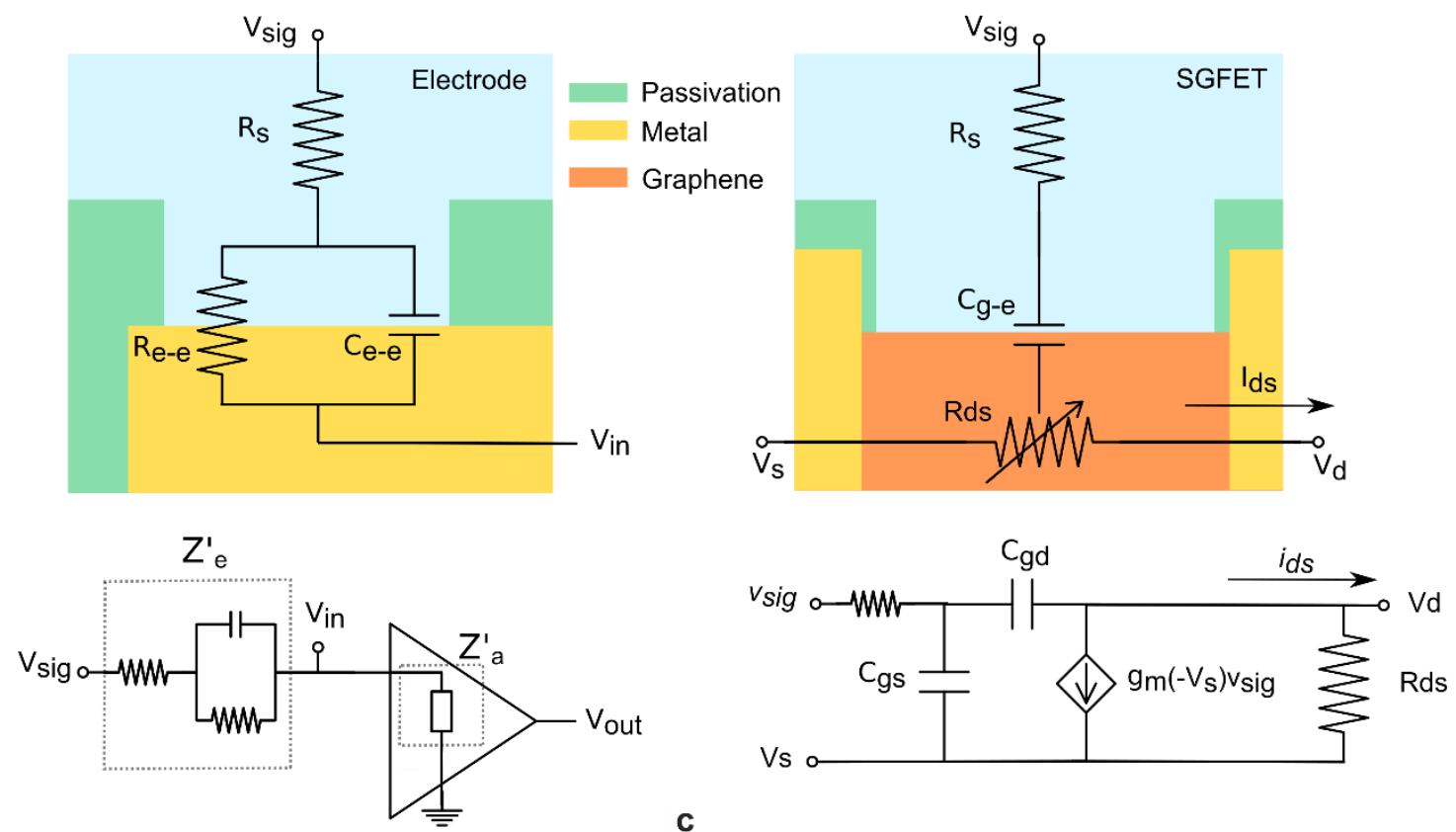

b
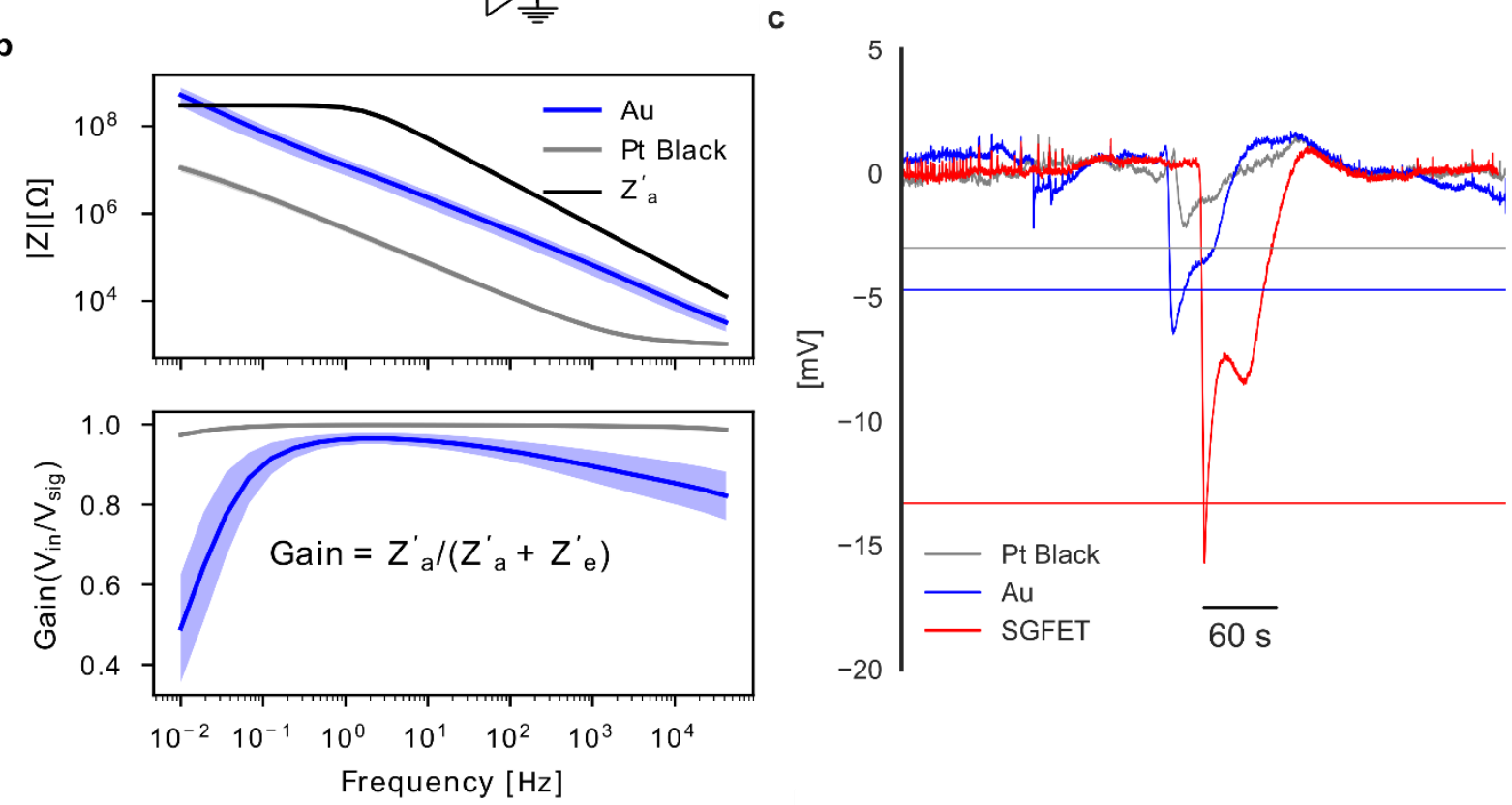

197 Fig. 4 | Microelectrode and gSGFET recording modes and signal filtering. a, Cross-sectional view and 198 superimposed electric equivalent circuit models of a recording electrode and a gSGFET. For an electrode, the 199 electrode-electrolyte interface, is modelled simply as a capacitor and a resistor in parallel $\left(\mathrm{R}_{\mathrm{e}-\mathrm{e}}, \mathrm{C}_{\mathrm{e}-\mathrm{e}}\right)$. The 200 voltage divider formed by $Z_{e}^{\prime}$ and $Z_{a}^{\prime}$, the effective electrode and amplifier impedance, respectively, 201 determines $V_{i n}$, the voltage at the input of the amplifier. $\mathrm{R}_{\mathrm{s}}$ represents the electrolyte resistance. In the case of a 202 gSGFET, $V_{\text {sig }}$ modulates the graphene channel resistance $\left(R_{d s}\right)$ by field-effect through the gate capacitance $\left(C_{g}\right.$ $203 e$ ), which results in current variations $\left(i_{d s}\right)$ proportional to the transconductance value at the bias point, plus the 204 voltage signal (which is mostly negligible for small amplitude electrophysiological signals), as seen in the 205 small signal model. b, Mean and standard deviation of the impedance module (experimental data) of the 50 $206 \mu \mathrm{m}$ diameter gold (blue) and platinum black (grey) microelectrodes together with the amplifier impedance $207\left(Z_{a}^{\prime}\right)$ and calculated voltage gain $\left(V_{i n} / V_{\text {sig }}\right)$ for each microelectrode type. c, Recordings of a CSD event for 208 each type of microelectrodes and a gSGFET. Horizontal lines represent the mean value of CSD amplitude. 


\section{Mapping CSD with gSGFETs}

210 As an example of the potential of gSGFET technology we mapped the propagation of CSD events

211 using a 4x4 epicortical gSGFET array and compare the signals with what is observed in conventional

212 high-pass filtered recordings (Fig. 5a-b). The recording of the whole CSD event with the gSGFET

213 array reveals that while the onset of the negative shift is similar for all gSGFETs, there is much more

214 variety in the subsequent recovery, with some transistors exhibiting a second negative shift with

215 higher amplitude than the first one. This effect can also be observed in the last frames (corresponding

216 to $80 \mathrm{~s}$ and $90 \mathrm{~s}$ ) of the spatial maps of gSGFET recordings (Fig. 5b) where recovered and still

217 depressed brain areas coexist. Importantly, this information is lost in conventional microelectrode

218 recordings, where only the CSD onset is observed due to the high pass filter in the recording

219 electronics. The following results are referred to a sample of 10 CSDs collected from two different

220 subjects in the somatosensory cortex: we found that the mean duration of CSD events is $47.24 \pm 7.65$

$221 \mathrm{~s}$ and a speed of propagation of $7.68 \pm 1.35 \mathrm{~mm} / \mathrm{min}$, in agreement with the literature defining CSDs

222 as infraslow brainwaves. Further details of the propagation analysis and results (Fig. S7 can be found

223 in the Supplementary Information.

224 It is known that under physiological conditions there is a normal neurovascular response defined by

225 vasodilatation and increased $\mathrm{rCBF}$ due to spreading depolarization that causes spreading hyperemia ${ }^{9}$.

226 However, most studies on CSD neurovascular coupling have been performed with mapping

227 techniques for the rCBF while electrical activity is measured only at two sites with glass

228 micropippetes $^{5}$. Here, taking advantage of the gSGFET technology, we designed an experiment in

229 which we could simultaneously map both variables. Fig. 5c provides further evidence of the

230 spreading depolarization and hyperemia neurovascular coupling. We used a non-contact, wide-field

231 technique, laser speckle contrast imaging (LSCI) ${ }^{36}$, which consists in the measurement of the

232 fluctuations of the laser speckle pattern produced by coherent light when it is scattered from an

233 illuminated object. In vivo, the presence of dynamic scatterers, mainly moving red blood cells, allows

234 to image variations of $\mathrm{rCBF}^{37}$. Experimentally, a craniotomy was performed in a Wistar rat and a

235 continuous-wave temperature controlled laser diode and a camera were mounted to image a wide

236 area inside which an epicortical 16-channel gSGFET array was placed. After $5 \mathrm{mM} \mathrm{KCl}$

237 administration, CSD was induced, which was followed by an increase in rCBF that slowly returned

238 (4-5minutes) to basal values. Importantly, gSGFETs did not hamper rCBF measurements, as metal

239 microelectrodes do, and thus allow the measurement of rCBF and electrophysiological signals

240 simultaneously over the same area.

241 To further illustrate the potential of gSGFET technology and taking advantage of the design 242 versatility offered by this technology, we performed in vivo experiments with intracortical probes 243 consisting of a linear array of 15 gSGFETs spanning the entire depth of the cortex (Fig. 6a). From 244 either the ordered recording or the spatiotemporal voltage map (Fig. 6b), it can be seen how CSD 245 occurs in the whole cortex depth. These results highlight the capability of gSGFET technology to 246 reveal the rich pattern of infraslow signals in the cortex; in this particular case, a transition from a 247 superficial long depolarization to a shorter one preceded and followed by a hyperpolarization in the 248 deeper layers is clearly observed. The origin of such depth-dependent effect is not well understood 
249 and will be the target of further investigations, taking advantage of the demonstrated capability of 250 gSGFET technology to monitor ISA with high spatial resolution.
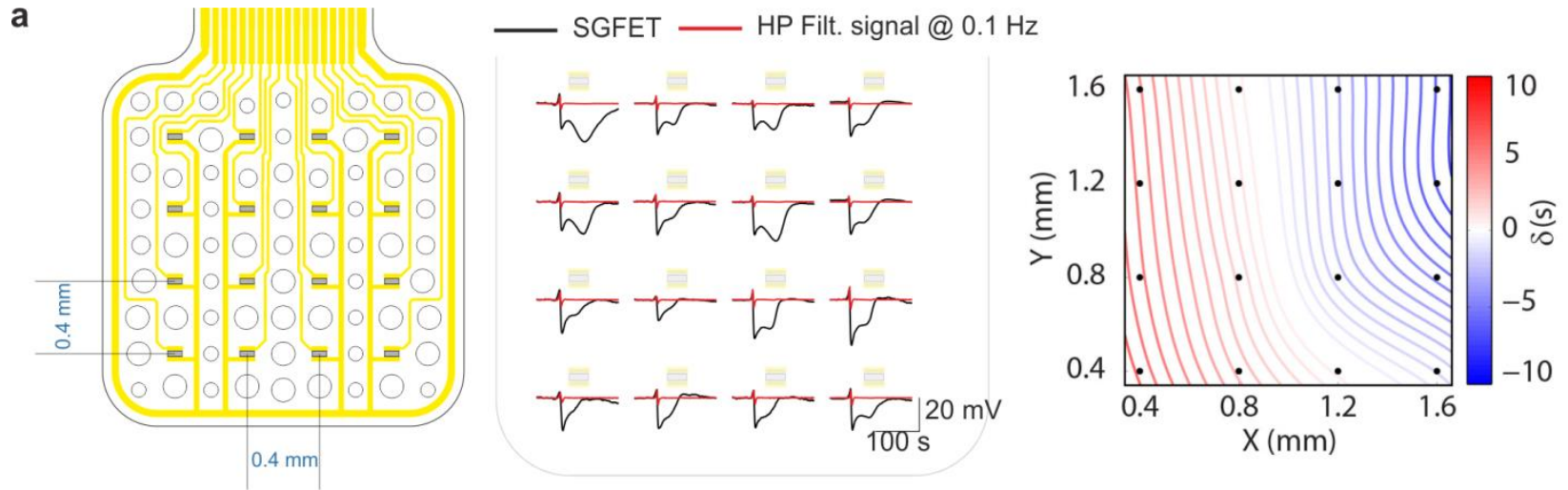

b

$[\mathrm{mm}]$

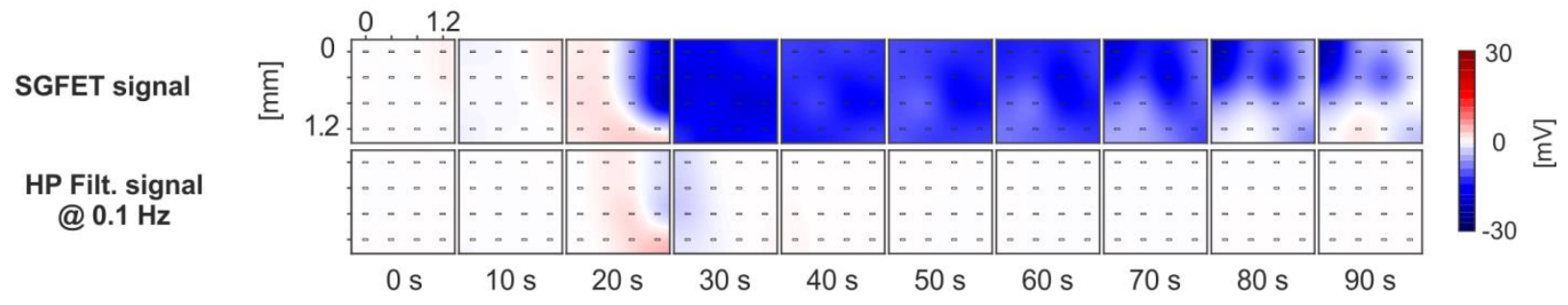

C
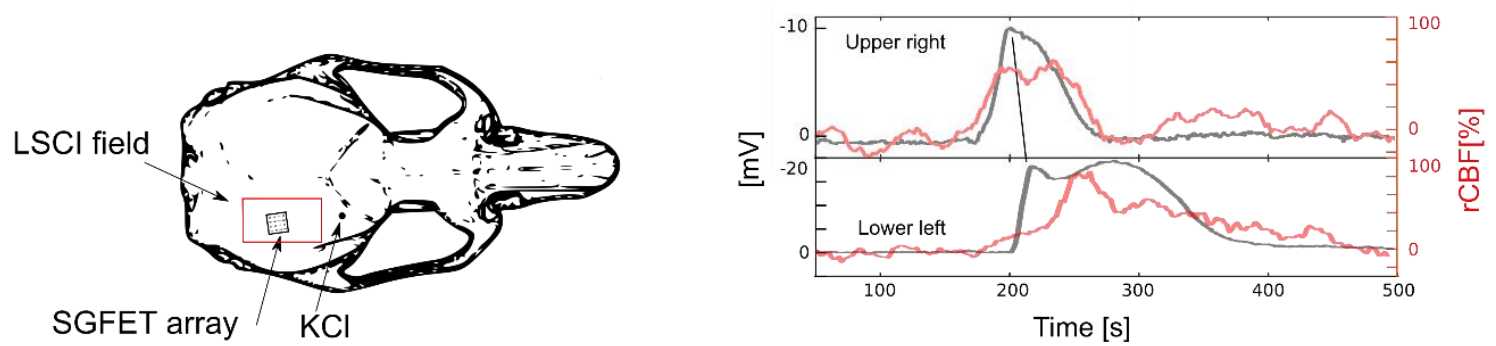

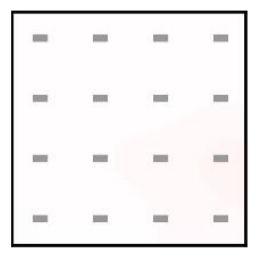

$t=150 s$

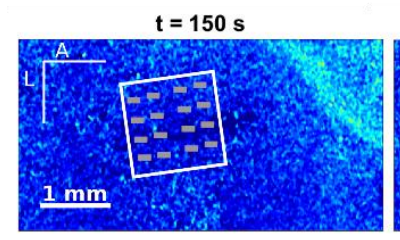

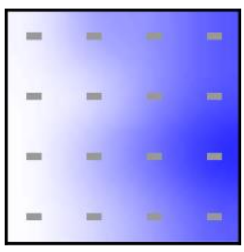

$t=200 s$

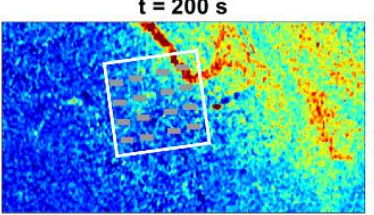

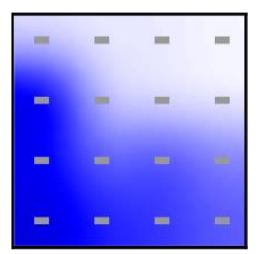

$\mathbf{t}=\mathbf{3 0 0 \mathrm { s }}$

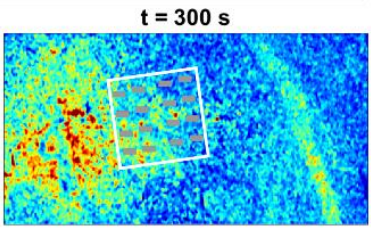

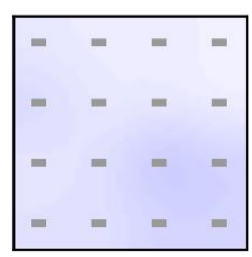
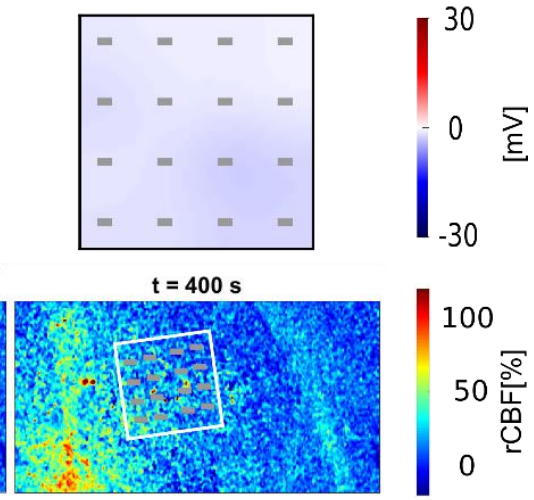

100

$50 \stackrel{\frac{2}{2}}{\frac{1}{0}}$

253 Fig. 5 | Graphene transistor arrays enable mapping of cortical spreading depression in vivo. a, Infralow

254 frequency signals recorded by a $4 \times 4,400 \mu \mathrm{m}$ grid spacing, gSGFET array (black lines) during the occurrence

255 of a CSD event and contour plot of the spatiotemporal course of the CSD. b. Interpolated spatial voltage maps

256 showing the propagation of the same CSD event as measured by the $4 \mathrm{x} 4$ epicortical gSGFET array. a,b High

257 pass filtered recordings at $0.1 \mathrm{~Hz}$ (red lines in $\mathbf{a}$ and bottom spatial voltage maps in $\mathbf{b}$ are included to illustrate

258 the loss of signal information in conventional microelectrode recordings. c, Schematic of a rat skull depicting

259 the laser speckle contrast imaging field-of-view and the position of the gSGFET array. Time evolution of the

260 upper right and lower left graphene microtransistors as well as the regional cerebral blood flow at the same 
261 position. Color maps represents the extracellular voltage as measured by the gSGFET array (top) and the 262 relative cerebral blood flow (bottom) at a given time after the induction of a CSD by $5 \mathrm{mM} \mathrm{KCl}$.

a

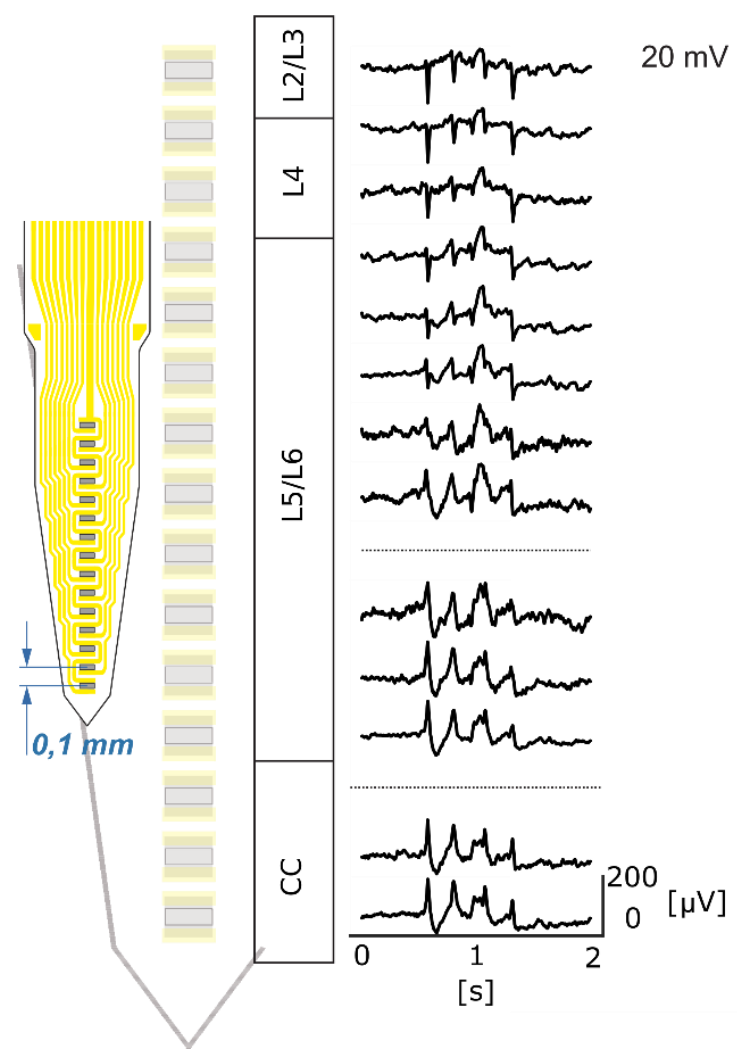

b
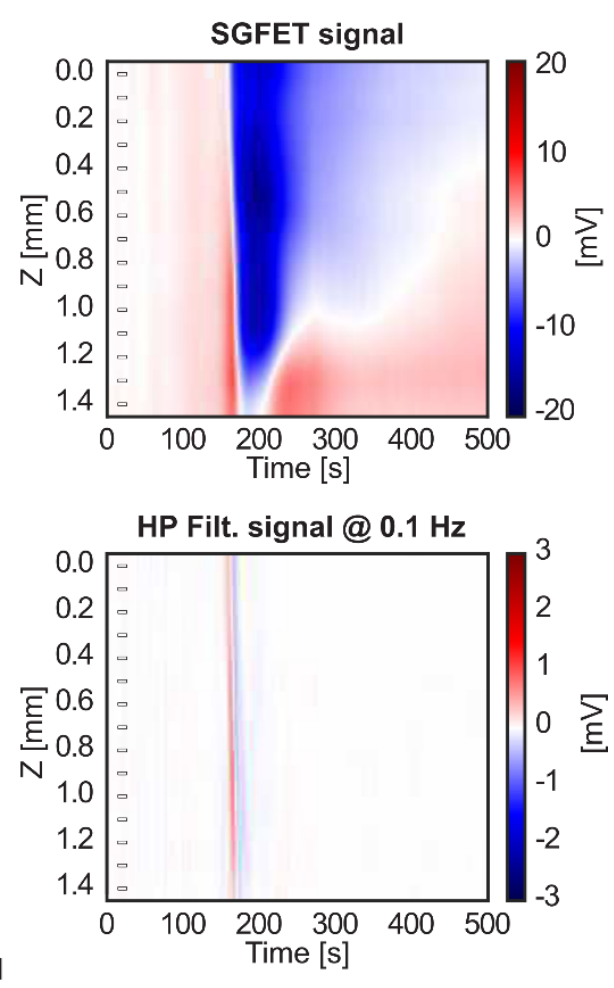

263

264 Fig. 6 | Depth profile of infralow-frequency voltage variations induced by cortical spreading

\section{Outlook}

271 In this work we show that gSGFETs can record neural signals in a wide electrophysiological 272 bandwidth, including infralow $(<0.1 \mathrm{~Hz})$ frequencies. There are two main reasons that explain this 273 unique capability: the direct DC-coupling, in contrast to standard passive electrodes, and the 274 excellent electrochemical stability. Making use of such capabilities, gSGFET technology opens the 275 possibility to map infraslow oscillations with high spatiotemporal resolution (epicortically and 276 intracortically) which can lead to a better understanding of the brain regions where ISA is initiated, 277 its propagation to other areas and clarify the interplay of different cellular types, which are yet poorly 278 understood $^{1,2,38}$. The physiological implications of ISA to a wide range of brain functions as also its 279 pathophysiological consequences and contribution to neural disorders would benefit chronic use of 280 gSGFET technology potentially leading to the discovery of new therapies. Additionally, gSGFETs 281 can help in determining ISA relation with higher frequency signals ${ }^{16,39}$ and contribute to a better 282 understanding of the genesis of local field potentials ${ }^{40}$ and of cortical wave propagation features ${ }^{41,42}$. 
283 Also, obtained data demonstrate that gSGFETs and LSCI can be used together to map 284 electrophysiological signals and $\mathrm{rCBF}$ therefore allowing improved characterization of the 285 neurovascular coupling of ISA. In the particular case of CSDs, gSGFET technology emerges as a 286 potential clinically relevant tool to help determine the relation of CSDs to neural disorders such as 287 migraine, malignant stroke, subarachnoid and intracranial haemorrhage, and traumatic brain injury. If 288 the challenges of translating gSGFET technology to the clinics are surpassed, a first direct 289 application could be for CSD intraoperative monitoring since there is evidence that CSD can occur 290 during neurosurgical procedures ${ }^{43}$. In summary, our work strongly suggests that gSGFET arrays are 291 ideal candidates to fill the gap of a large-scale, high-spatiotemporal recording technology that covers 292 a wide electrophysiological bandwidth in a potentially fully implantable, nontoxic, clinical-scale 293 device. We believe that the demonstrated capabilities of graphene microtransistors constitute a 294 considerable advance in electrophysiological recording technology which could lead to

295 breakthroughs in brain function understanding as well as in clinic diagnosis and treatment.

\section{References}

2971 Hughes, S. W., Lörincz, M. L., Parri, H. R. \& Crunelli, V. in Progress in Brain Research Vol. 193 (eds Eus J. W. Van Someren et al.) 145-162 (Elsevier, 2011).

2992 Mitra, A. et al. Spontaneous Infra-slow Brain Activity Has Unique Spatiotemporal Dynamics $300 \quad$ and Laminar Structure. Neuron, doi:https://doi.org/10.1016/j.neuron.2018.03.015.

3013 Lecci, S. et al. Coordinated infraslow neural and cardiac oscillations mark fragility and 302 offline periods in mammalian sleep. Science Advances 3, e1602026 (2017).

3034 Mitra, A., Snyder, A. Z., Tagliazucchi, E., Laufs, H. \& Raichle, M. E. Propagated infra-slow intrinsic brain activity reorganizes across wake and slow wave sleep. Elife 4 (2015). Hiltunen, T. et al. Infra-slow EEG fluctuations are correlated with resting-state network dynamics in fMRI. Journal of Neuroscience 34, 356-362 (2014).

3128 Chung, D. Y. \& Ayata, C. in Primer on Cerebrovascular Diseases (Second Edition) (eds Leopold, D. A., Murayama, Y. \& Logothetis, N. K. Very slow activity fluctuations in monkey visual cortex: implications for functional brain imaging. Cerebral cortex 13, 422-433 (2003).

3149 Dreier, J. P. The role of spreading depression, spreading depolarization and spreading ischemia in neurological disease. Nat Med 17, 439-447 (2011). Dreier, Jens P. \& Reiffurth, C. The Stroke-Migraine Depolarization Continuum. Neuron 86, 902-922, doi:https://doi.org/10.1016/j.neuron.2015.04.004 (2015).

Lauritzen, M. et al. Clinical relevance of cortical spreading depression in neurological disorders: migraine, malignant stroke, subarachnoid and intracranial hemorrhage, and traumatic brain injury. Journal of Cerebral Blood Flow \& Metabolism 31, 17-35 (2011). 
Hartings, J. A. et al. Direct current electrocorticography for clinical neuromonitoring of spreading depolarizations. Journal of Cerebral Blood Flow \& Metabolism, $0271678 X 16653135$ (2016).

Dreier, J. P. et al. Recording, analysis, and interpretation of spreading depolarizations in Cerebral Blood Flow \& Metabolism, $0271678 X 16654496$ (2016).

\section{Kovac, S., Speckmann, E.-J. \& Gorji, A. Uncensored EEG: The role of DC potentials in} neurobiology of the brain. Progress in Neurobiology, doi:https://doi.org/10.1016/j.pneurobio.2018.02.001 (2018).

\section{Vanhatalo, S., Voipio, J. \& Kaila, K. Full-band EEG (FbEEG): an emerging standard in} electroencephalography. Clinical Neurophysiology 116, 1-8, doi:http://dx.doi.org/10.1016/j.clinph.2004.09.015 (2005).

Vanhatalo, S. et al. Infraslow oscillations modulate excitability and interictal epileptic activity in the human cortex during sleep. Proceedings of the National Academy of Sciences of the United States of America 101, 5053-5057 (2004).

Hofmeijer, J. et al. Detecting Cortical Spreading Depolarization with Full Band Scalp Electroencephalography: An Illusion? Frontiers in Neurology $\mathbf{9}$, doi:10.3389/fneur.2018.00017 (2018).

Ayata, C. \& Lauritzen, M. Spreading depression, spreading depolarizations, and the cerebral vasculature. Physiological reviews 95, 953-993 (2015).

Stensaas, S. S. \& Stensaas, L. J. Histopathological evaluation of materials implanted in the cerebral cortex. Acta Neuropathologica 41, 145-155, doi:10.1007/bf00689766 (1978).

Li, C. et al. Evaluation of microelectrode materials for direct-current electrocorticography. Journal of neural engineering 13, 016008 (2015).

Hartings, J. A. et al. Direct current electrocorticography for clinical neuromonitoring of spreading depolarizations. Journal of Cerebral Blood Flow \& Metabolism 37, 1857-1870 (2017).

Nelson, M. J., Pouget, P., Nilsen, E. A., Patten, C. D. \& Schall, J. D. Review of signal distortion through metal microelectrode recording circuits and filters. Journal of neuroscience methods 169, 141-157 (2008).

Stacey, W. C. et al. Potential for unreliable interpretation of EEG recorded with microelectrodes. Epilepsia 54, 1391-1401 (2013).

Kuzum, D. et al. Transparent and flexible low noise graphene electrodes for simultaneous electrophysiology and neuroimaging. Nature communications 5, 5259 (2014).

Deneux, T. et al. Accurate spike estimation from noisy calcium signals for ultrafast threedimensional imaging of large neuronal populations in vivo. Nature Communications $\mathbf{7}$, 12190, doi:10.1038/ncomms12190 https://www.nature.com/articles/ncomms12190\#supplementary-information (2016).

Fang, H. et al. Capacitively coupled arrays of multiplexed flexible silicon transistors for longterm cardiac electrophysiology. Nature biomedical engineering 1, 0038 (2017). 
$361 \quad 27$

362

363

36428

365

36629

367

$368 \quad 30$

369

$370 \quad 31$

371

$372 \quad 32$

373

374

$375 \quad 33$

376

377

$378 \quad 34$

379

$380 \quad 35$

381

382

$383 \quad 36$

384

385

$386 \quad 37$

387

$388 \quad 38$

389

390

39139

392

39340

394

39541

396

$397 \quad 42$

398

39943

400

Heremans, P. et al. Mechanical and electronic properties of thin-film transistors on plastic, and their integration in flexible electronic applications. Advanced Materials 28, 4266-4282 (2016).

8 Hess, L. H., Seifert, M. \& Garrido, J. A. Graphene transistors for bioelectronics. Proceedings of the IEEE 101, 1780-1792 (2013).

Kim, B. J. et al. High-Performance Flexible Graphene Field Effect Transistors with Ion Gel Gate Dielectrics. Nano Letters 10, 3464-3466, doi:10.1021/nl101559n (2010).

Kostarelos, K., Vincent, M., Hebert, C. \& Garrido, J. A. Graphene in the Design and Engineering of Next-Generation Neural Interfaces. Advanced Materials 29 (2017).

Benno, M. B. et al. Mapping brain activity with flexible graphene micro-transistors. $2 D$ Materials 4, 025040 (2017).

Hébert, C. et al. Flexible Graphene Solution-Gated Field-Effect Transistors: Efficient Transducers for Micro-Electrocorticography. Advanced Functional Materials 28, 1703976, doi:doi:10.1002/adfm.201703976 (2018).

Chen, S., Liu, Y. \& Chen, J. Heterogeneous electron transfer at nanoscopic electrodes: importance of electronic structures and electric double layers. Chemical Society Reviews $\mathbf{4 3}$, 5372-5386 (2014).

4 Brownson, D. A. \& Banks, C. E. The electrochemistry of CVD graphene: progress and prospects. Physical Chemistry Chemical Physics 14, 8264-8281 (2012).

5 Brownson, D. A. C., Munro, L. J., Kampouris, D. K. \& Banks, C. E. Electrochemistry of graphene: not such a beneficial electrode material? RSC Advances 1, 978, doi:10.1039/c1ra00393c (2011).

Valdes, C. P. et al. Speckle contrast optical spectroscopy, a non-invasive, diffuse optical method for measuring microvascular blood flow in tissue. Biomedical optics express 5, 27692784 (2014).

Boas, D. A. \& Dunn, A. K. Laser speckle contrast imaging in biomedical optics. Journal of biomedical optics 15, 011109 (2010).

38 Shibata, M. \& Suzuki, N. Exploring the role of microglia in cortical spreading depression in neurological disease. Journal of Cerebral Blood Flow \& Metabolism 37, 1182-1191, doi:10.1177/0271678x17690537 (2017).

Mitra, A. \& Raichle, M. E. How networks communicate: propagation patterns in spontaneous brain activity. Phil. Trans. R. Soc. B 371, 20150546 (2016).

Herreras, O. Local field potentials: myths and misunderstandings. Frontiers in neural circuits 10, 101 (2016).

401 


\section{Acknowledgments}

403 This work is part of the requirements to achieve the $\mathrm{PhD}$ in Electrical and Telecommunication 404 Engineering at the Universitat Autònoma de Barcelona, and it was funded by the European Union's 405 Horizon 2020 research and innovation programme under Grant Agreement No. 696656 (Graphene 406 Flagship). This work has made use of the Spanish ICTS Network MICRONANOFABS partially 407 supported by MINECO and the ICTS 'NANBIOSIS', more specifically by the Micro408 NanoTechnology Unit of the CIBER in Bioengineering, Biomaterials \& Nanomedicne (CIBER409 BBN) at the IMB-CNM. E. d. C. thanks to Spanish Ministerio de Economía y Competitividad for the 410 Juan de la Cierva postdoctoral grant IJCI-2015-25201. T.Du acknowledges support from Fundació 411 CELLEX Barcelona, Ministerio de Economía y Competitividad /FEDER (PHOTODEMENTIA, 412 DPI2015-64358-C2-1-R), the "Severo Ochoa" Programme for Centres of Excellence in R\&D (SEV413 2015-0522) and the Obra social "la Caixa" Foundation (LlumMedBcn).

\section{Author contributions}

415 E.M.C. did most of the fabrication and characterization of the gSGFET arrays, contributed to the 416 design and performance of the in vivo experiments, analyzed the data and wrote the manuscript. X.I. 417 designed the neural probes and fabricated the microelectrode arrays. A.B.C. contributed to the 418 fabrication and characterization of the gSGFET arrays. M.D. performed the in vivo experiments. 419 P.G., C.H., J.B. and E.P.A. contributed to the growth of the CVD graphene. E.P.A., E.dC. and 420 J.M.dC.S. contributed to the transfer of graphene. E.P.A., E.dC.G. and G.R. contributed to the 421 characterization of CVD graphene. J.M.A. contributed to the fabrication of the custom electronic 422 instrumentation and development of a python-based user interface. A.C. contributed to the 423 propagation analysis. T.Dr., E.V. and T.Du. contributed to the in vivo measurements and analysis of 424 cerebral blood flow. M.D., M.S.V., A.G.B, R.V. and J.A.G. participated in the design of the in vivo 425 experiments and thoroughly reviewed the manuscript. A.G.B. contributed in the design and 426 fabrication of the custom electronic instrumentation, development of a custom gSGFET python 427 library and in the analysis of the data. All authors read and reviewed the manuscript.

\section{Competing interests}

429 The authors declare no competing interests.

\section{Methods}

\section{Graphene growth and characterization}

432 Graphene layers were grown by Chemical Vapor Deposition (CVD) using one of the following 433 procedures: a) A lamp-heated rapid thermal CVD equipment from Jipelec and $25 \mu \mathrm{m}$ thick, $99.8 \%$ 434 metal basis copper foil provided by AlfaAesar have been employed. Prior to graphene CVD, copper 435 foils were sequentially cleaned in acetic acid and acetone, and finally rinsed in isopropyl alcohol 436 (IPA). Sample dimensions were $6 \times 5 \mathrm{~cm}^{2}$. Growth processing conditions consisted in 10 minutes at $437750{ }^{\circ} \mathrm{C}, 200 \mathrm{sccm} \mathrm{H}$ plus 5 minutes at $800{ }^{\circ} \mathrm{C}, 25 \mathrm{sccm} \mathrm{CH}_{4} / 200 \mathrm{sccm} \mathrm{H}$. b) Chemical vapour 438 deposition on a $4.5 \times 7 \mathrm{~cm}^{2}$ copper foil (Alfa Aesar, annealed, Coated). Prior to the growth, the copper 439 foil was electropolished during $5 \mathrm{~min}$ at a fixed current density of $62 \mathrm{~mA} / \mathrm{cm}^{-2}$ in a solution 440 containing $\mathrm{H}_{2} \mathrm{O}(1 \mathrm{~L})+\mathrm{H}_{3} \mathrm{PO}_{4}(0.5 \mathrm{~L})+$ ethanol $(0.5 \mathrm{~L})+$ isopropanol $(0.1 \mathrm{~L})$ and urea $(10 \mathrm{~g})$. Then 441 the copper foil was loaded in a planar quartz tube $(1600 \times 60 \mathrm{~mm})$ heated by a three zone oven. A first 442 annealing step at $1015^{\circ} \mathrm{C}$ under a $400 \mathrm{sccm}$ argon flow at $100 \mathrm{mbar}$ during $1 \mathrm{~h}$ was followed by a 15 - 
443 min growth step at 12 mbar under a gas mixture of $1000 \mathrm{sccm}$ argon, $200 \mathrm{sccm}$ hydrogen and $2 \mathrm{sccm}$ 444 of methane. The sample was then cooled down under a $400 \mathrm{sccm}$ argon flow by removing the quartz 445 tube from the oven. A complete Raman characterization was performed on each sample using a 446 Witec spectrograph (Fig.S1a-d). Raman maps of 30x30 $\mu^{2}$ were registered with a spatial resolution 447 lower than $1 \mu \mathrm{m}^{2}$ (using a 50x objective). We used a $488 \mathrm{~nm}$ excitation wavelength to minimize the 448 cooper substrate luminescence signal. The laser power was kept below $1.5 \mathrm{~mW}$ to avoid sample

\section{0 gSGFET array fabrication and characterization}

451 Four-inch silicon wafers were used as a support to build the devices. First, a $10-\mu \mathrm{m}$-thick polyimide 452 layer (PI-2611, HD MicroSystems) was spin-coated to be used as substrate and hard-baked at $350^{\circ} \mathrm{C}$ 453 to complete the imidation process. Graphene transistors were fabricated in a sandwich-like structure. 454 For that, a first layer of metal (Ti/Au, 10/100 nm) was evaporated and defined in a standard lift-off 455 process using the image reversal photoresist AZ5214E (Clariant GmbH, Germany). Then, single456 layer graphene was transferred by electrochemical delamination ${ }^{1}$. After removing the PMMA 457 protection layer, the graphene active areas were defined by means of an oxygen-based reactive ion 458 etching (RIE). A second metal layer (Ni/Au, 20/200nm) was evaporated and defined in a similar 459 standard lift-off process avoiding the use of ultrasounds in order to maintain graphene integrity. SU-8 460 (SU-8 2005, MicroChemCorp., USA) a permanent epoxy-based negative photoresist was used to 461 passivate the metal leads while defining the graphene channel and metal contacts. Finally, the 462 polyimide substrate was structured in a deep-RIE process using the thick AZ9260 positive 463 photoresist (Clariant $\mathrm{GmbH}$, Germany) as an etching mask. Polyimide probes were directly peeled 464 off from the wafer and placed in a zero insertion force (ZIF) connectors to be interfaced with our 465 custom electronic instrumentation. Current-voltage measurements of graphene transistors were 466 performed in common gate mode with a fixed drain-source voltage $\left(\mathrm{V}_{\mathrm{DS}}=50 \mathrm{mV}\right)$ varying the gate467 source voltage $\left(\mathrm{V}_{\mathrm{GS}}\right)$ vs. a $\mathrm{Ag} / \mathrm{AgCl}$ reference electrode in $0.01 \mathrm{M}$ PBS solution. Steady-state was 468 ensured by acquiring only after time derivative of $1 \mathrm{~s}$ of current is below $5 \mathrm{e}-7 \mathrm{~A} / \mathrm{s}$. The total leakage 469 current was measured for the whole array and corresponds to the sum of the individual leakage 470 currents of all transistors in the array. The frequency response of the transconductance was measured 471 by applying a sum of sinusoidal signals at the electrolyte solution through the reference electrode and 472 by measuring the modulation of the drain current. Measures were split into two bands, low 473 frequencies $(\approx 0-10 \mathrm{~Hz})$ in which drain-source current was simultaneous acquired for all transistors in 474 a probe, and high frequencies $(10 \mathrm{~Hz}-30 \mathrm{kHz})$ in which each transistor was recorded individually.

\section{Microelectrode array fabrication and characterization}

476 The flexible microelectrode array was fabricated in polyimide in a very similar process. Here, a $477 \mathrm{Ti} / \mathrm{Au}(20 / 200 \mathrm{~nm})$ metal layer was evaporated on a $10 \mu \mathrm{m}$-thick polyimide-covered four-inch silicon 478 wafer to define the metal tracks and the microelectrodes, while a second polyimide layer $(2 \mu \mathrm{m}$ thick $)$ 479 was used as the passivation layer. Two subsequent etching steps were used to open, firstly, the 480 microelectrode active areas and, secondly, to structure the polyimide in order to define the probe 481 geometry which is the same as in Illa et. al. ${ }^{2}$. Platinum black was deposited in some electrodes by 482 constant polarization amperometry. A voltage of $-0.2 \mathrm{~V}$ against a $\mathrm{Ag} / \mathrm{AgCl}$ reference electrode was 483 applied during $15 \mathrm{~s}$. Impedance spectra were measured against a $\mathrm{Ag} / \mathrm{AgCl}$ reference electrode using a 484 Solartron SI 1260 equipment (Solarton analytical, UK) with $20 \mathrm{mV}$ signal amplitude.

\section{In vivo recordings}

486 Eight adult male Wistar rats (225-375 g) were used in this study. Animals were deeply anaesthetized 487 with isoflurane (4\% induction, 1-3\% maintenance) and all pressure and incision points were 488 infiltrated with local anesthetic lidocaine. Once under the surgical plane of anesthesia, animals were 
489 transferred to a stereotaxic frame with body temperature constantly monitored and maintained at $49037^{\circ} \mathrm{C}$ by means of a thermal blanket. A craniotomy and durotomy were performed on the left 491 hemisphere over either the primary somatosensory (S1, AP: X to Y from bregma) or visual (V1) 492 cortices in order to record with surface or penetrating probes, respectively. Additionally, a 493 craniotomy and durotomy were performed over the prefrontal cortex to topically administer $5 \mathrm{mM}$ $494 \mathrm{KCl}$ to induce cortical spreading depression. A Ag/ $\mathrm{AgCl}$ electrode was inserted in temporal muscle 495 and used as reference. A custom electronic instrumentation was used which provides the current-to496 voltage conversion and the bias control for each channel. The instrumentation splits the recorded 497 signals into two bands with different gains: low-pass filtered $\left(<0.16 \mathrm{~Hz}, 10^{4}\right.$ gain) and band-pass 498 filtered $\left(0.16 \mathrm{~Hz}<\mathrm{f}<160 \mathrm{kHz}, 10^{6}\right.$ gain). The low-pass filtered signals and bias control is managed 499 by a data acquisition system (National Instruments USB-6353), while the band-pass filtered signals 500 were directly acquired by a commercial electrophysiological recording system consisting of a 501 programmable gain amplifier (Multichannel Systems, $\mathrm{GmbH}$ ) and digitizer interface (CED 1401 and 502 Spike2 software, Cambrigde Electronic Design, UK). LPF band was sampled at $1 \mathrm{~Hz}$ and BPF at 5 $503 \mathrm{kHz}$. Prior to the beginning of the recordings, the transfer curve of the gSGFET was measured in situ 504 to determine the best bias point, generally around $-0.1 \mathrm{~V}$ of the CNP. For the electrode and transistor 505 comparison experiment, a custom Simulink model was used to simultaneously measure graphene 506 transistors through an adapted g.HIamp biosignal amplifier(g.tec medical engineering $\mathrm{GmbH}$, 507 Austria) while microelectrodes were recorded using an g.USBamp (g.tec medical engineering GmbH, 508 Austria). The same $\mathrm{Ag} / \mathrm{AgCl}$ reference electrode was used by both amplifiers and signals were 509 sampled at $5 \mathrm{kHz}$.

\section{Laser speckle contrast imaging}

511 For the measurement of the regional cerebral blood flow $(\mathrm{rCBF})$, a laser speckle contrast imaging 512 (LSCI) system was used which consists of a continuous-wave temperature-controlled laser diode 513 (785 nm, Thorlabs, Germany) for homogenous full-field illumination and a charge-coupled device 514 camera (sc640-120fm, Basler, Germany), with an exposure time of $5 \mathrm{~ms}$, which captures the diffused 515 light scattered from the imaging area. The speckle contrast was calculated for the predefined region 516 of interest (ROI) at each pixel in temporal domain over 100 frames, to ensure good signal-to-noise 517 ratio. The statistics of different noise sources $^{3}$ was accounted for when calculating the speckle 518 contrast. Speckle contrast was then related to a $\mathrm{rCBF}$ index (BF) as reported in ${ }^{4,5}$. Finally, the

519 relative blood flow $(\triangle \mathrm{rCBF})$ was calculated as:

$$
\Delta r C B F=\frac{B F-B F_{B}}{B F_{B}} * 100[\%]
$$

521 where $B F_{B}$ corresponds to the basal regional blood flow.

\section{Data Analysis}

523 All data were analyzed using Python 2.7 packages (Matplotlib, Numpy and Neo) and the custom 524 library PyGFET (https://github.com/aguimera/PyGFET). The conversion of the recorded current 525 signals (LPF and BPF) to a voltage signal was performed by summation and interpolation in the in 526 vivo measured transfer curve of the corresponding gSGFET at the bias point. For visualization 527 purposes microelectrode recordings were filtered (band-stop, 48-52 Hz) and down sampled at $300 \mathrm{~Hz}$. 528 For the propagation analysis, the baseline of the signal was estimated as the mean value of the signal 529 until the positive deflection. We defined the onset of the CSD as the onset of the negative shift and 530 detected it using a threshold (Fig. S7a). We defined the WaveTime of each wave as the mean time of 531 the triggers detected in the 16 transistors and constructed a TimeLagMatrix containing time lags for 532 each channel computed with respect to the WaveTime (Fig. S7c). We interpolated the known time 
533 lags with a thin-plate smoothing spline technique. The velocity of the propagation has been estimated 534 computing the gradient of the TimeLagMatrix on the grid $^{6}$. To determine the direction of the waves, 535 a vector starting at the point with higher negative delay (leader of the propagation) and pointing to

536 the one with the highest positive delay (follower of the propagation) was transformed into polar 537 coordinates to obtain the angle (Fig. S7b).

\section{Methods References}

5391 de la Rosa, C. J. L. et al. Frame assisted H[sub 2]O electrolysis induced H[sub 2] bubbling 540 transfer of large area graphene grown by chemical vapor deposition on $\mathrm{Cu}$. Applied Physics $541 \quad$ Letters 102, 022101-022104 (2013).

5422 Illa, X., Rebollo, B., Gabriel, G., Sánchez-Vives, M. V. \& Villa, R. in SPIE

543 Microtechnologies. 951803-951803-951806 (International Society for Optics and Photonics).

$5443 \quad$ Valdes, C. P. et al. Speckle contrast optical spectroscopy, a non-invasive, diffuse optical method for measuring microvascular blood flow in tissue. Biomedical optics express 5, 2769$5462784(2014)$.

5474 Boas, D. A. \& Dunn, A. K. Laser speckle contrast imaging in biomedical optics. Journal of $548 \quad$ biomedical optics 15, 011109 (2010).

5495 Bandyopadhyay, R., Gittings, A., Suh, S., Dixon, P. \& Durian, D. J. Speckle-visibility spectroscopy: A tool to study time-varying dynamics. Review of scientific instruments 76, $551093110(2005)$.

5526 Capone, C. et al. Slow Waves in Cortical Slices: How Spontaneous Activity is Shaped by 553 Laminar Structure. Cerebral Cortex, 1-17 (2017). 